\title{
A Named Data Networking Architecture Implementation to Internet of Underwater Things
}

\author{
Qi Zhao \\ University of California, Los Angeles \\ Los Angeles, CA \\ qi.zhao@cs.ucla.edu
}

\author{
Zheng Peng \\ The City College of New York \\ New York, NY \\ zpeng@ccny.cuny.edu
}

\author{
Xiaoyan Hong \\ The University of Alabama \\ Tuscaloosa, AL \\ hxy@cs.ua.edu
}

\begin{abstract}
The Internet of Underwater Things (IoUT) advances our ability in exploring oceans, lakes and rivers through multiple communication technologies that connect stationary and mobile nodes underwater, at the surface and in the sky. However, characteristics such as low data rate, long propagation delay, energy-constraint, mobility and sparsity of underwater communications remain as major challenges to the potential benefits that IoUT can bring to data availability and data sciences. This paper aims to further explore how the Named Data Networking (NDN), a future Internet architecture, addresses the challenges of IoUT and can be adapted to potentially provide a more secure, simplified, and efficient implementation of IoUT. The IoUT network and application semantics are aligned with the datacentric communication model of NDN, which enables operators to deploy and configure networks more easily, and developers to focus more on "things" and data. The paper starts from introducing new challenges in IoUT and then illustrating in detail with simple examples to show how to employ NDN architecture to IoUT and how to enable additional functionalities required by IoUT.
\end{abstract}

\section{KEYWORDS}

Internet of Underwater Things, Named Data Networking, Datacentric

\section{INTRODUCTION}

The ocean is becoming a busy place as human beings are deploying more instruments and devices into the seas for better understandings of the planet's aquatic environments. In the past decade, many underwater communication and networking architectures have been introduced $[5,7,24]$, and a number of prototypes, testbeds and platforms have been implemented [23, 25]. A typical Internet of Underwater Things (IoUT) can be a three layer networked system that involves (see Fig. 1): (i) a large number of underwater sensors (e.g. CTD and ADCP), acoustic positioning systems (e.g. LBL), autonomous underwater vehicles (AUVs), unmanned surface vehicles (USVs), surface buoys and unmanned aerial vehicles (UAVs); (ii) acoustics communications, local RF links, cellular, or satellite links connecting these Things; (iii) network topology be single hop

Permission to make digital or hard copies of all or part of this work for personal or classroom use is granted without fee provided that copies are not made or distributed for profit or commercial advantage and that copies bear this notice and the full citation on the first page. Copyrights for components of this work owned by others than ACM must be honored. Abstracting with credit is permitted. To copy otherwise, or republish, to post on servers or to redistribute to lists, requires prior specific permission and/or a fee. Request permissions from permissions@acm.org.

WUWNET'19, October 23-25, 2019, Atlanta, GA, USA

(C) 2019 Association for Computing Machinery.

ACM ISBN 978-1-4503-7740-9/19/10 .\$15.00

https://doi.org/10.1145/3366486.3366506 or ad-hoc multiple hops, static or mobile or even intermittent, or cloud-based; (iv) users can be nearby sensors and AUVs, surface buoys and ships, or on shore. The main tasks of IoUT, then, can be summarized as collecting data, sharing data, controlling actuation, and with possible data analytics in between.

Unfortunately, despite the many efforts in researches and developments, data sharing and message distribution are still a challenging process at the core of IoUT. For examples, there are different network architectures, no universal and straightforward user interfaces, and ubiquitous data consumers and diversified data types. Such issues complicate the communication solutions of IoUT over IP. This paper focuses on those significant networking challenges unmet by existing networking solutions based on IP and host-tohost communication model. It will discuss how the Named Data Networking (NDN) architecture can mitigate the challenges in IoUT [10].

NDN has emerged as a new network architecture for efficient, reliable and safe data sharing. In this paper, we argue that NDN provides an effective solution for IoUT. Instead of organizing a network based on host-to-host connections, NDN builds a network based on named data and user interests on the named data. In NDN, data adopts a certain, usually hierarchical, naming convention. Users express their interests on data by the names to the network; the network then returns the matching data. In such communication model, no host addresses are involved. This unique feature is very suitable for IoUT applications in which heterogeneous networks are involved, some of which may not be of conventional designs. NDN offers the users a universal access to data despite the technologies in use, locations of the users or the data, nor the need for network address hierarchy or the addressing mechanism. As shown in Fig. 1, users can be from a variety of locations, e.g., an office on shore, a research ship at sea, or an AUV under the water. The same query can be issued to retrieve the named data of interests, irrespective of user locations too. This architecture makes data sharing and distribution much easier, especially when the network structure of the underwater sensor network, the surface ad-hoc network, and the Internet are very different.

The related work has studied a few aspects of NDN in underwater communication and networking. But the multitude of advantages to IoUT have not been fully discussed, nor been developed into practical networked systems and underwater simulations. This paper will show how NDN addresses the underwater communication, networking and IoUT system challenges, and when implement, what and how networking functions are to be adapted.

The main advantages of using NDN in IoUT include: (i) NDN helps reducing the burden in managing IP addresses and NATs by replacing them with unified data-name based APIs. This leads to a 


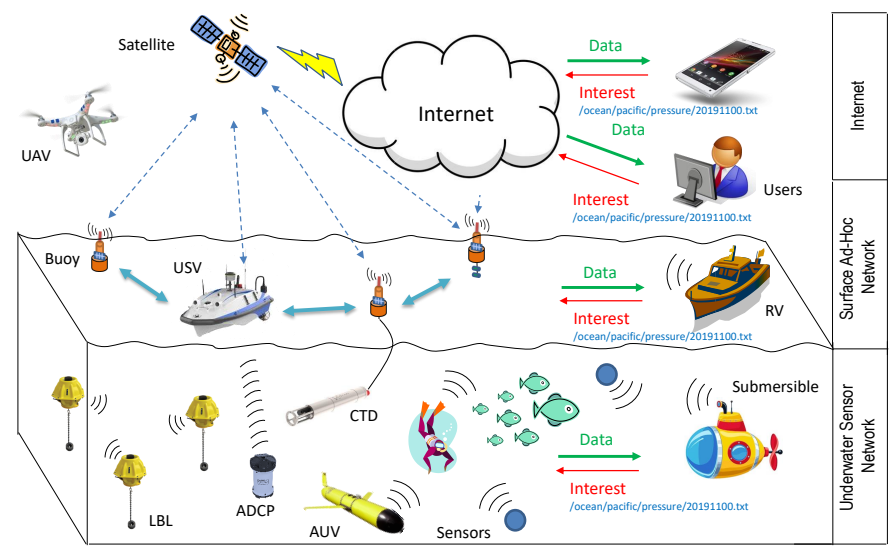

Figure 1: Internet of Underwater Things

significant shift of the requirement for the domain users, i.e., from technology (especially networking) knowledge back to their expertise on domain data. (ii) The NDN architecture brings efficiency via in-network caching in balancing multiple factors of bandwidth, latency, energy and mobility. (iii) NDN enables security binding to data itself, unifying and easing the management of access and trust of the data and network operations.

The rest of the paper is organized in the following order. Section 2 gives an overview of NDN and related work. Section 3 presents the discussions on the challenges and the advantages from NDN. Section 4 describes the implementation of the NDN components in IoUT; while Section 5 introduces additional IoUT functionality to be enhanced by NDN architecture. Section 6 concludes the paper.

\section{BACKGROUND AND RELATED WORK}

\subsection{The Internet of Underwater Things}

The concept of Internet of Underwater Things emerged ca. 2012 [8], which describes a world-wide network of smart and interconnected underwater devices. Since 2014, FP7 SUNRISE Project started to build an open infrastructure to facilitate the development of IoUT technologies [25]. The project provides a unified interface for the heterogeneous resources (acoustic modems, underwater sensors, AUV, etc) offered by the testbeds of the SUNRISE federation. Another notable effort toward the realization of IoUT is the SEANet project [7]. The objective is to develop a new generation of programmable platforms and a networking testbed for IoUT. Its hadware platform is based on ARM Cortex-A9 and FPGA, while the network protocol stack is based on the TCP/IP architecture with reconfiguration capabilities.

\subsection{NDN Overview}

NDN changes the semantics of network service from delivering the packet to a given destination address to fetching data identified by a given name. NDN builds a network based on named data and user interests in the named data, instead of a network based on hostto-host connections [10]. In NDN, data has a certain hierarchical naming convention. The data providers publish the data names. The users can express their interests to the network. If the matching data exists in the network, the users will receive the data in return. Data caching (Content Store) is a built-in component at the NDN routers. In such communication model, no host addresses are involved.

NDN uses two packet types, namely, the Interest packet sent by the data consumer and the Data packet sent from the provider or a cache. The NDN forwarder, located at each router, first checks its Content Store to see if there is any Data matches the Interest with exactly the same name or name prefix. The found Data will be forwarded to satisfy the Interest. Otherwise, the forwarder checks its Pending Interest Table (PIT), which implements the forwarding across the entire NDN data plane by recording each pending Interest and its incoming interface(s), and removing the Interest after the matching Data is received or upon a timeout event, to decide the next step. If found in PIT, its incoming interface will be added. If not, this Interest will be recorded in PIT and then forwarded based on the Forwarding Information Base (FIB) and Forwarding Strategy. The forwarding interface will be determined by Interest name lookup in FIB with longest prefix match. Similarly, when a Data arrives at the NDN router, PIT lookup will be performed to verify that whether this Data is desired by any pending Interest. Then, the Data will be forwarded according to the interfaces recorded by the matched PIT entries, or discarded otherwise. Satisfied PIT entry removal and Data caching will also be performed before forwarding the Data. Note that the lifetime stored with each Interest entry in the PIT prevents the potential Interest flooding attack. Meanwhile, the policy of one Interest requesting at most one Data ensures the consumer-driven flow balancing.

NDN secures the data itself directly by requiring data producers to cryptographically sign the binding of every data chunk and its name [10]. This network-layer security ensures integrity and enables determination of data provenance independently of how or where it is obtained. It also allows consumers to reason about whether a public key owner is allowed to access a specific piece of data in a specific context.

\subsection{Underwater NDN}

In the past few years, several preliminary studies have been published regarding underwater NDN architecture. In [16], Martin et al. proposed a routing protocol to create adaptive and robust forwarding tables based on data categorization and NDN. In [13], Kuai et al. proposed a preliminary study on the feasibility of using the NDN architecture to construct an underwater monitoring systems. The authors concluded that a NDN based method can achieve high user interest satisfaction rate. Partridge et al. studied NDN in large detached underwater sensing arrays [22]. The goal was to evaluate how well NDN could handle the communication needs. The authors focused on a network that covers thousands of square kilometers with underwater sensors that were $15 \mathrm{~km}$ away from each other. Additionally, Bouk et al. also investigated NDN for underwater acoustic networks. The authors described an underwater NDN architecture, proposed a naming scheme for UNDN and launched simulations to evaluate how transmission range and node density affect the user interest satisfaction rate and delay [4]. A recent work [35] studied the energy consumption of an underwater NDN architecture with direct transmissions and two-hop transmissions. Simulations were conducted in both shallow water and deep water 
settings. The authors concluded that the node distance, replay position and communication frequency would play an important role in selecting direct or two-hop transmissions for energy efficient underwater wireless communications.

\section{IoUT CHALLENGES}

The IoUT vision faces many challenges with current IP-dependent networking solutions. In this section, we discuss the challenges and the ways NDN can mitigate each challenge.

\subsection{Complex Communication Solutions}

IoUTs render a tremendous diversity in communication technologies. The current underwater communication systems mainly rely on acoustic communication technologies [6]. The large propagation delay of acoustic waves and high bit error rate of the underwater acoustic channel still hinder communication performance severely [8]. In recent years, various new underwater communication technologies, such as underwater optical communications and Magneto-inductive (MI) communications, emerged to enhance the performance in terms of throughput, delay and reliability [3]. The hardware frontends employing these underwater communication technologies may have various radios, network interfaces and links, potentially with different addressing schemes, IP subnets and mapping mechanisms between network-layer packets and application data.

On the other hand, in IoUT, identification and tracking technologies are quite different from traditional wireless networks or wired IoT. Radio Frequency Identification (RFID) is commonly used in wireless networks and IoT for tracking. However, IoUT has more complex settings depending on the type of things to be tracked and the specific characteristics of such things [8]. Different tracking tasks may require different devices employed with different tracking technologies for various detection ranges and sensor types, which further complicates the underwater communication scenario in terms of addressing these things.

Ultimately, the access to all connected devices in IoUT requires either a local middleware to manage associated interoperability or a remote cloud service to handle the data retrieval and distribution. As a result, the middleware developers would have onerous task of handling the data flow among a wide variety of devices and services. Developing such overlay on top of host-to-host communication model is non-trivial, let alone maintaining it in a dynamic scenario, where all devices, network interfaces and communication channels may change constantly. Thus, further problems emerge. One problem is that managing the changes requires intensive resources and operations, which is limited in IoUT. The other problem is that such complexity hinders the usability and operability as the users are normally not experts in networking.

Apparently, lots of efforts have been given to enable the underwater devices to communicate with existing protocol suite even before working with the things themselves. The above problems need to be solved to simplify the implementation of the mapping between network interfaces and underwater devices to provide a more feasible underwater network with even higher performance.

With the NDN architecture, the complexity in the diversity of the communication solutions and addresses of the data can be eased by named-data standardized interfaces. The NDN communication interfaces are request-response based APIs. On the other hand, domain scientists have the expertise on data they desire and can be involved in naming the data and requesting needed data. Such interfaces simplify the development and deployment for applications.

\subsection{Energy Limitation}

Many of the underwater instruments rely on batteries to operate. Due to the space limit in the waterproof compartment, these underwater instruments have limited operation life time. Combined with the fact that replacing batteries could be a logistic nightmare in many underwater applications, the IoUT design inherently needs to pay additional attention to the energy efficiency.

The underwater acoustic communications are power-hungry. For example, a typical value of Wi-Fi transmitter power consumption is $100 \mathrm{~mW}$, whereas the power consumption of an acoustic modem is $35-150$ watts [32], 2-3 orders of magnitude in difference. Of the acoustic transmission power, much of it is to combat the path loss, which is largely affected by the communication distance. The attenuation of acoustic signals underwater can be estimated as [30]:

$$
10 \log A(l, f)=k \cdot 10 \log l+l \cdot 10 \log a(f)
$$

where $l$ is the distance between the source and destination, $k$ is the spreading factor ( $1 \leq k \leq 2$ in underwater environment), and $a(f)$ is the absorption coefficient determined by the acoustic channel frequency $f$. Eq. 1 shows that the energy loss grows faster than the increase of the distance.

The energy efficiency of the NDN architecture manifests in several ways. First, under the NDN architecture, a user no longer needs to communicate directly with a remote sensor. Instead, the user can get the needed information from a nearby node via caches. In other words, NDN can reduce the amount of communications by leveraging the storage capacity of the network. In this way, a large amount of energy can be saved. Second, with a shorter communication distance, the same transmission power leads to a much higher SNR, which usually translates to lower BER (i.e., higher reliability). Therefore, less energy will be wasted in re-transmissions. Third, even for a mesh network that the nodes only communicate with its direct neighbors on the grid, NDN architecture can reduce the number of communications needed to flood the Interest by using the broadcast links. Therefore, adapting the NDN architecture will bring energy efficiency to IoUTs.

\subsection{Bandwidth and Mobility}

IoUT devices are limited by the scarce communication resources due to the high cost of the devices and the extremely low data rates achievable by the underwater links. Mobility, due to the widely used mobile devices, gliders, AUVs or surface ships, further challenges the communications among them in underwater explorations. Researches in underwater networking and communications have strove greatly to improve the data transfer capabilities. When communications is not sufficient or possible, one way in practice can be storing the data and delivering it as stored data at the end of the missions. However, IoUTs will bring the underwater related research fields into a Big Data era. The ability of generating and 
collecting data from IoUT devices can easily surpass the communication capability when transferring them. Methods have to be developed to solve the issues relating to bandwidth and mobility. NDN based IoUT brings two advances. First, the integrated in-network storage helps store data pieces for later transmissions in the cases of bandwidth efficiency or mobility; Second, the named-data communication model allows the scarce communication resources to be used only for the data of interests on-demand. In general, NDN architecture offers the flexibility in-network management in terms of leveraging bandwidth, mobility, storage, and data retrieval.

\subsection{Reliability}

Underwater reliable data transfer is critical yet extremely challenging. On the one hand, the underwater acoustic communication suffers from very high bit error rate, due to the special characteristics of the underwater acoustic channel, such as the temporal and spatial dynamics, long multi-path spread, limited bandwidth, and severe frequency-dependent attenuations. For example, the bit error rate of RF communications is in the range of $10^{-5} \sim 10^{-6}$, whereas the bit error rate of acoustic communications is about $10^{-2} \backsim 10^{-1}$ [20]. On the other hand, many underwater applications require data to be correctly received in sequence without any loss. In terrestrial networks, this is usually handled by TCP. However, the long propagation delay in underwater environment makes end-to-end feedback based protocol, such as TCP, ineffective [6].

NDN architecture can address the reliability issue using a hopby-hop based approach. Typically, the data retrieval is based on Interest-Data exchanges between one-hop neighbors: an Interest packet is sent to a neighbor, and a Data packet with the matching name is returned. The reliable data transfer can be achieved by repeating it for each hop. The mechanisms of Interest flooding and caching can reduce the need to repeat the process or retransmission in multiple hops. For underwater acoustic networks, it is shown in recent literature that the hop-by-hop based reliable data transfer can significantly reduce the end-to-end delay [17, 18, 34]. Many of these methods adopt coding algorithms to protect both the Interest and Data packets, hence can be seamlessly incorporated into the underwater NDN architecture.

\subsection{Security Limitations}

IoUT is sensitive to several security issues: (i) The data can be private to some applications, needing confidentiality; (ii) Most underwater devices like sensors are not as cheap as those in landbased IoTs since they always equipped with sophisticated sensing and measuring tools. They are also designed to be waterproof and pressure-resistant. These devices are mostly unattended in areas not having security guide, leading to potentially physical device tampering. A secure network and communication system is necessary to prevent data breach and device tampering. However, the security approaches used in current Internet applications are not a good fit for IoUT. For solutions in TCP/IP networks, the security process mostly happens at the ends of a communication session, which is brittle in IoUT networks with heterogeneous devices and multiple communication technologies and channels used simultaneously on one device. For solutions that employ cloud services for device communication to centralize the security to cloud side, it basically violates the trend of pushing computation to edge in IoUT network with loosely coupled devices. For example, an underwater sensor equipped with multiple communication interfaces, i.e., acoustic communication, optical communication, etc. Each of them has different protocol stacks and corresponding security solutions, which complicates the entire implementation.

Furthermore, TCP/IP based security solutions cannot natively handle some requirements of IoUT, such as, representing identity of the devices with an unique name, management of the provenance of the data, authenticating trusted elements during communication, etc. Overall, the traditional network security solutions are neither efficient nor effective to solve the security challenge in IoUT. NDN's data-centric security built within the request-response primitive offers authentication for the requested data to bind to the requested name. This native security property simplifies the security implementation in diverse IoUT netowork scenarios.

\section{NDN ARCHITECTURE TO IOUT}

In this section, we introduce the core NDN architecture detail and the implementation of NDN for IoUT to address the challenges mentioned in Section 3.

\subsection{NDN Protocol and Architecture Implementation}

In NDN, communication is driven by data consumers (receivers), through requesting data by sending out Interest packets that carry a name or name prefix of the desired data. On the other hand, data producers (senders) generate Data packets with names that can be retrieved by NDN network through Interest packets with the same name. Once the Interest reaches a node that has the requested data, the node will return a Data packet that contains both the name and the content, together with a signature by the producer's key which binds the two. The Data packet follows the reversed path taken by the Interest to get back to the requesting consumer. NDN's data-centric security is the foundation for maintaining security property while traveling among various network environments via the secured request-response primitive. It also enables applications to enjoy the benefits of in-network storage without putting efforts on securing the intermediate nodes any more. Such a communication model of securely retrieving named data naturally matches the semantics of IoUT applications. It also allows the applications to define own data names out of domain knowledge. For the example of monitoring and measurement application in IoUT, users can simply use the Interest-Data request-response primitive to retrieve named Data from underwater sensors over the NDN network.

To deal with various underlying communication technologies that are compatible with the TCP/IP protocol stack, a practical way is to create a NDN overlay as the middleaware. The overlay will handle the functionalities of mapping network interfaces to underwater devices, managing the name space of the intended data, and managing the security of the communication. Additional efforts are needed as well: (i) a Layer 2 configuration to ensure traffic flows among heterogeneous IoUT subnets; (ii) IP address and port number assignment management for network connectivity; (iii) secure data collection and forwarding. 


\subsection{IoUT Data and Naming}

NDN uses meaningful names for the application data, instead of end hosts' addresses, at the network layer. The design of the naming schemes then becomes critical when developing NDN-based applications. Though NDN does not restrict the naming scheme for applications as long as they are unique and meaningful to the application, it is highly recommended to follow certain naming conventions as well as data payload formats to enable a universal format at the network and application layers. Here, a hierarchical, human-readable name is used for illustration in the case of underwater water temperature data responding to a surface node: "/Surface/Sensor-397/Temperature/1907250202". The name is constructed with three parts: (i) a root prefix representing the scope ("/Surface"); (ii) middle components representing the IoUT devices (“.../Sensor-397/..."); (iii) tail components representing the specific data (“.../Temperature/201907250202").

Such naming structure provides more flexibility for data grouping and properly forwarding. Also, Interest packet can retrieve desired data using that name. "/Surface/Sensor-397/Temperature" included in the Interest packet requests all the temperature data collected by sensor 397; but adding ". . ./1907250202" to that Interest packet will retrieve all the temperature data collected by sensor 397 at that specific time. Specifically, the sensor 397 responds to the corresponding name accordingly.

The types of marine application data include time series data, location data, 2-dimension images, channel properties data (time series arrays), videos, real-time, etc. Many of the types can use similar naming conventions as those used in wire-line based IoT as well as the Interest-Data request-response primitive to retrieve the named Data from IoUT nodes. However, one type of data, such as control commands, are initiated proactively by a controller based on management needs, and they should be delivered promptly. In addition, there is a need in some cases that the commands be issued without identifying the specific target and creating a communication link explicitly, especially when dealing with a set of nodes. With NDN, users can use Interest packets to express the control commands. The mechanism is to carry the control commands at the Parameters field in the Interest packet and send the Interest to a device without a prior Interest on the command [1]. Such Interests contain the target node name prefix and the corresponding commands need to be delivered to target nodes, e.g., "/Surface/Move-left/50m". The Name should specify the unique command and parameters if any. Meanwhile, the command Interest contains the information to authenticate the issuer of the command and the command with the parameters. Encryption can also be added if needed. In summary, NDN brings and enhances the security, convenience and efficiency for IoUT command communication.

\subsection{Data-Centric Security}

Security is a critical challenge for IoUT in both securing the data from breach and protecting the node from being hacked. In IoUT, each Data packet is signed when generated by nodes with binding the name and the payload of the data cryptographically. Both name and the payload can also be encrypted, depending on the sensitivity of the application. The information of the signing key is stored in the KeyLocator at the Signature field in the Data packet
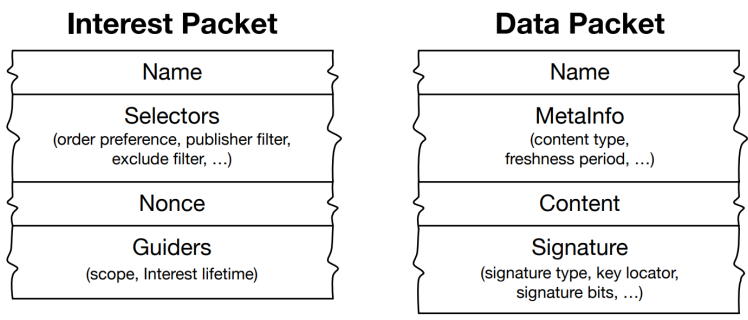

Figure 2: NDN packets construction [10].

as shown in Figure 2. Various cryptographic mechanisms, such as asymmetric RSA and ECDSA signatures [31], are explored recently to provide the feasibility of handling different application scenarios. In addition, applications can control access to data via encryption and distribute keys as encrypted NDN data, limiting the data security perimeter to the context of a single application [10]. Such securing the data at network layer provides the foundation for securing the routing protocols against different type of attacks including spoofing and tampering. Specifically for IoUT, it helps the network to meet the security requirements of being independent of the specific communication technology used for transmitting data. NDN enables the data in IoUT to maintain the security property while traveling among various network environments. Besides, NDN makes it possible to freely store the data in the in-network caches or persistent data storage in IoUT without exact efforts on securing any intermediate node. More details of this advantage will be introduced in later sections.

At last, NDN's use of multi-path forwarding, together with the adaptive forwarding strategy module, mitigates prefix hijacking because routers can detect anomalies caused by hijacks and retrieve data through alternate paths [37], which enhances the security, reliability and performance of IoUT simultaneously.

\subsection{Routing and Forwarding}

Routing and forwarding in NDN are purely based on the names. Therefore, (i) there is no address exhaustion problem any more since the namespace is unlimited; (ii) there is no NAT traversal problem since there is no addresses at all; (iii) address assignment and management are no longer needed. For IoUT, it means applications can operate directly on NDN packets and the data inside.

Existing underwater routing protocols can be revised and used for data publishers to advertise the name prefixes in NDN architecture. For example, some sensing data can be published by the sensors at the sea floor, while the users can be at the surface. The depth-based underwater routing protocols, such as DBR [36] and HydroCast [21], can be used by these sensors for better data forwarding paths. Similarly, if location information is a part of the names, geo-routing based underwater routing protocols, such as VBF [33] and FBR [11], can be employed to help populating the FIBs for these data.

\subsection{In-network Storage}

NDN's foundation of securing the binding of the name and the data directly enables applications to enjoy the benefits of in-network storage without putting efforts on securing the intermediate nodes any more. Firstly, routers in NDN network can opportunistically 
cache the received Data packets in its Content Store and use them to satisfy future Interests or facilitate local recovery. Since NDN treats storage and network channels identically in terms of data retrieval, either static or dynamic (e.g., realtime monitoring) contents can benefit from caching [10]. Individual devices can adjust their cache size and caching policy based on their status dynamically. Moreover, NDN supports persistent data repositories that provide larger and long-term storage for data, without requiring any extra engineering effort to work. NDN's in-network storage also provides different privacy protection than IP networks. Malicious users can examine the packet header or payload to find out who the data receiver is from the in-network cache in IP network while it is much harder in NDN since there is no destination address in NDN packets.

IoUT applications can leverage all types of in-network storage at the same time. For example, underwater sensors can transfer collected data immediately to its nearby deployed repository since it is costly to equip large storage on those sensors. Then, surface users can retrieve data from the repository instead of sensors whenever the available bandwidth is sufficient without causing any network congestion. Besides, it has been proved that the in-network caching improves the efficiency of data dissemination effectively by caching the most recent forwarded data packets to satisfy the same requests in the future. When collected data is requested by multiple users, in-network opportunistic caches will help with efficient data forwarding by reducing the transmission hops and propagation distance. Due to the sparsity and mobility characteristics of IoUT, network connectivity may not always be maintained and the communication must be delay-tolerant, where NDN's in-network storage can perfectly fit in.

\section{ADDITIONAL IoUT FUNCTIONALITIES}

An internet of underwater things based on NDN is a distributed system building on top of the NDN architecture. It has additional functionalities to handle data repositories, system maintenance, access control and application adaptations. In this section, we show how NDN architecture can help in these areas.

\subsection{Data Aggregation}

It is desirable that the IoUT devices perform data aggregation within its subsystem after establishing the network connectivity and then deliver the aggregated data to some permanent storage nearby within the network. A subsystem can be a mesh underwater sensor networks, coordinated AUVs, etc.. IoUT applications at the ocean surface can retrieve the stored data at a later time. Associated with the aggregation is the light-weight pre-processing on the raw data The reason for such operation is because IoUT devices normally generate a huge amount of data constantly and they often lack of sufficient storage and processing power to store and process the collected raw data. Such in-network caching also improves the efficiency and reliability of the data retrievals. Moreover, the data analysis operations can be pushed to the water edge - the intermediate gateway nodes or even permanent storage nodes, to increase the efficiency of the system since the data can be processed as early as possible after being collected.
The data-centric semantics and the naming of NDN fit nicely for the data aggregation requirements of IoUT. With NDN, IoUT applications decide the naming convention for the data collected from IoUT devices. The intermediate gateway nodes or permanent storage nodes can retrieve the data periodically based on the naming convention in two ways. One way uses Interest packet to retrieve the name specified data in Section 4.2. The other uses a NDN based publish-subscribe (pub-sub) framework. In the framework, underwater devices are the data publishers, which announce the name prefixes with Interests so as to "publish" their data to the NDN network. The applications are data subscribers, which can later request the desired data via sending Interest packets with corresponding names. The applications only have to make sure the published data state be refreshed in time so to learn newly published data.

\subsection{Synchronization}

In IoUT, communication between multiple collaborated devices is another main application scenario. In this case, data pieces are to be shared among them via multiple data producers under the same name prefix, e.g., "/ocean/pacific/santa-monica". Based on the Interest-Data exchange primitive, NDN Dataset Synchronization (NDN Sync) [14, 41] provides the dataset synchronization ability among multiple devices in the network with reliable multicast communication. This is totally different from the traditional cloud-based synchronization mechanisms because NDN Sync is decentralized with no server involved.

To achieve such synchronization, the following problems have to be resolved: (i) name the dataset and encode it to represent the local state; (ii) notify other peers the change of the shared dataset; (iii) detect the difference between local dataset and shared one, and update local one if necessary. For example, ChronoSync [41] adopts a monotonically increasing sequential data naming convention and encodes the local dataset into a crypto digest data structure, which represents the local state. Then, peers can keep synchronized by exchanging the Sync Interest and Reply packets. Specifically, they periodically send out Sync Interest packets with their local dataset state and, at the meantime, keep track of the difference between their own local dataset states and the Sync state received from Sync Interest packets. If the Sync state is newer, corresponding updates will be performed. If the local dataset state is newer, a Sync Reply packet will be sent out to notify everyone about the new data. Otherwise, it remains at the steady stage.

NDN Sync can be adapted to implement data sharing and collaborative operation among IoUT devices in a local network environment. For example, multiple collaborative underwater sensors in a common area can quickly synchronize their own collected data to share their partial knowledge to everyone. In addition, NDN Sync is especially suitable for the information sharing and dissemination for tracking and localizing underwater mobile devices, navigation, and task coordination because they often experience disruptive acoustic communication environments and power saving where intermittent connectivity and dynamic network topology exists. NDN Sync allows the short-lived underwater devices to discover missing data from other peers via fast data synchronization to achieve efficient message delivery. 


\subsection{Bootstrapping and Discovery}

Device management mechanisms, specifically bootstrapping and discovery are difficulty for either the network operators or users once the devices are deployed underwater. In traditional IP networks, device bootstrapping and discovery usually involve complicated and tedious operations, which is even worse when dealing with different networks and communication medias. However, in NDN, bootstrapping and discovery can be simplified by using the Interest-Data request-response primitive to deliver three key configurations for the devices: i) a proper name prefix for naming the produced data, ii) appropriate signing and encryption keys for encrypting the produced data, and iii) a trust anchor and decryption keys for consuming the retrieved data.

In NDN, requesting data without device addresses or even names are allowed so that IoUT devices can actually initiate an Interest packet with a meaningful name prefix containing sufficient information for identifying the device in the network. For example, "/santa-monica/discovery/sensor/serial=397" can be issued by an underwater sensor located near Santa Monica pier with serial number 397. Then, initial configuration will be performed after this Interest is received by a bootstrap controller. More realistic name (e.g., "/ocean/pacific/santa-monica/sensor-397") will be given to the device with further instructions (e.g., ". . /ON" and ". . /OFF"), if needed, and an appropriate trust anchor.

After the initial bootstrapping and device discovery, devices are named and installed with proper trust anchor. Other corresponding discoveries, such as device capability and service, can be performed as metadata exchange with the name prefix. For example, if the model information of the device is needed, ". ./sensor-397/make" or ". ./sensor-397/year" can be generated to satisfy that request. In a larger network, NDN Sync for names may be used as an efficient way to discover and manage newly joined or recovered devices.

\subsection{Access Control and Trust Management}

Align with NDN's data-centric security is the data-centric security management, with two key parts for IoUT implementation, namely, access control and trust management. Name-based Access Control (NAC) implements the content-based access control model in NDN [39]. It shows how to enable effective access control by using an additional access control namespace (parallel to the data namespace) to explicitly convey access control policy and distribute access control keys. NAC can be fully adopted to IoUT scenario to provide the access control functionality for users in IoUT. For example, the data owner Bob wants to encrypt his collected data from underwater sensors and grant the access control to Alice. Then, a content key (e.g., "/bob/data/NAC/CKEY") is needed from Bob for encryption and decryption of the data. Sensors can simple retrieve this key from Bob to encrypt collected data. To grant the access privilege to Alice, Bob needs to encrypt the content key with Alice's public key (e.g., "/alice/bob-data/NAC/EKEY") so that Alice should be able to decrypt the content key later for accessing the data by using her private key (e.g., "/alice/bob-data/NAC/DKEY"). More details can be found in $[39,40]$ for NDN IoUT implementation.

Trust management in IoUT is another critical issue of security since compromised trusts can potentially result in a data consumer treating valid data as malicious data or accepting false data. Worse cases can be a malicious user may be authorized by other users instead of administrators when an inappropriate key is used. Therefore, [38] presents a detailed design of the trust schema to provide the automation of data authentication and simplified key bootstrapping. The trust schema is a set of pre-defined rules that match data and key names and can be used to automate both authentications and signing processes. Specifically, IoUT system trust policies can be defined with a trust schema to specify the relations between data names and signing key names using the underwater domain knowledge for pattern matching on NDN names [27]. NDN provides the tools that automatically sign and verify the data according to that pre-defined trust schema. Such tools can even be integrated in to applications and dynamically adjusted later through client libraries. For example, a sensor belonged to Bob is configured with name prefix "/bob/santa-monica/monitoring" to publish data and the device key "/bob/sensors/397/key", which is signed with Bob's sensor root key. All monitoring data, such as temperature (e.g., "/bob/santa-monica/monitoring/temperature/time"), will be published under this name prefix and signed by that key. However, without the trust schema, the authentication has to be done manually by verifying the signature validity, which can be sophisticated for heterogeneous IoUT. In summary, trust schema is an helpful tool to handle the key discovery for both authenticating Data at consumer side and signing Data at producer side. It gives the operators and users the flexibility of defining the trust rules based on the network scenario to simplify the implementation.

\subsection{Application Adaptation}

Application adaptation, i.e., application support, is the major challenge for NDN development and deployment, which is also a big challenge for this NDN IoUT proposal. Even if the NDN has been deployed in underwater environment for IoUT devices with all functionalities discussed above, there is still the need to continuously add new applications, devices, and network components to this NDN IoUT architecture. A straightforward solution is to rewrite an application from scratch with full support of NDN, which may incur substantial rethinking, redesigning and rewriting, as seen from these early example applications [2, 9, 12, 29]. Another approach is to develop a proxy between transportation/network layer protocols and NDN [19, 26, 28]. Though no application change is needed for this approach, some architectural features will be sacrificed, e.g., translated NDN packets from TCP packets have to be associated to the specific TCP connection so that the caching across multiple TCP connections is lost.

To give the IoUT users' full benefits of NDN with minimum efforts, the work of placing the translation between application-level protocol and NDN is appealing [15]. With this approach, no application changes are involved so less development efforts would be incurred. In addition, such translation will generate more meaningful data names with application-level information than the previous proxy approach. In developing NDN IoUT applications, efforts can follow the detailed design and implementation framework, which includes application layer framing, application data unit defining, namespace design, data translation, application communication translation as well as security model adaptation [15]. 


\section{CONCLUSION}

This paper focused on exploring the potential opportunity of adapting NDN architecture to IoUT network environment in a systematical way. We briefly went over the existing challenges of IoUT based on the state-of-the-art underwater communication research and introduced ways that NDN architecture can address these challenges by its data-centric networking model. Then, we illustrated how to achieve the IoUT functionalities to solve the real problems in IoUT communication scenarios with NDN architecture, protocols and related mechanisms without using any IP. In such way, we aimed to shed light on an revolutionary direction for the future research and implementation opportunities of IoUT and IoUT applications. Our future works include more comprehensive and profound architectural design and framework implementation. Experimental examples and prototypical implementations for real IoUT scenarios are also needed for validation and evaluation.

\section{REFERENCES}

[1] 2018. NDN Specifications, Feature 4443. In Accessed fune 2019. https://redmine. named-data.net/issues/4443

[2] Alexander Afanasyev, Zhenkai Zhu, Yingdi Yu, Lijing Wang, and Lixia Zhang. 2015. The Story of ChronoShare, or How NDN Brought Distributed Secure File Sharing Back. In 2015 IEEE 12th International Conference on Mobile Ad Hoc and Sensor Systems. 525-530.

[3] Ian F. Akyildiz, Pu Wang, and Shih-Chun Lin. 2016. SoftWater: Software-defined networking for next-generation underwater communication systems. Ad Hoc Networks 46 (2016), 1 - 11

[4] Safdar Hussain Bouk, Syed Hassan Ahmed, and Dongkyun Kim. 2017. NDN Goes Deep: Foreseeing the Underwater Named Data Networks. In Proceedings of the Symposium on Applied Computing (SAC'17). 642-646.

[5] Mandar Chitre, Lee Freitag, Ethem Sozer, Shiraz Shahabudeen, Milica Stojanovic, and John Potter. 2006. An Architecture for Underwater Networks. In Proceedings IEEE OCEANS.

[6] Jun-Hong Cui, Jiejun Kong, Mario Gerla, and Shengli Zhou. 2006. Challenges: Building Scalable Mobile Underwater Wireless Sensor Networks for Aquatic Applications. IEEE Network (2006), 12-18.

[7] Emrecan Demirors, Jiacheng Shi, Anh Duong, Neil Dave, Raffaele Guida, Bernard Herrera, Flavius Pop, Guofeng Chen, Cristian Cassella, Sayedamirhossein Tadayon, Matteo Rinaldi, Stefano Basagni, Milica Stojanovic, and Tommaso Melodia. 2018. The SEANet Project: Toward a Programmable Internet of Underwater Things. In Proceedings of The Fourth Underwater Communications and Networking Conference (UComms).

[8] Mari Carmen Domingo. 2012. An Overview of the Internet of Underwater Things. Elsevier Journal of Network and Computer Applications (2012), 1879-1890.

[9] Peter Gusev and Jeff Burke. 2015. NDN-RTC: Real-Time Videoconferencing over Named Data Networking. In Proceedings of the 2Nd ACM Conference on Information-Centric Networking (ACM-ICN '15). 117-126.

[10] Van Jacobson, Diana K. Smetters, James D. Thornton, Michael F. Plass, Nicholas H. Briggs, and Rebecca L. Braynard. 2009. Networking Named Content. In Proceedings of the 5th international conference on Emerging networking experiments and technologies (CoNEXT'09).

[11] Josep Miquel Jornet, Milica Stojanovic, and Michele Zorzi. 2008. Focused Beam Routing Protocol for Underwater Acoustic Networks. In Proceedings WUWNet. San Francisco, CA, 75-81.

[12] Siham Khoussi, Davide Pesavento, Lotfi Benmohamed, and Abdella Battou. 2017. NDN-trace: A Path Tracing Utility for Named Data Networking. In Proceedings of the 4th ACM Conference on Information-Centric Networking (ICN '17). 116-122.

[13] Meng Kuai, Tarif Haque, Xiaoyan Hong, and Qiangyuan Yu. 2015. A Named-Data Networking Approach to Underwater Monitoring Systems. In Proceedings of the 10th International Conference on Underwater Networks and Systems (WUWNet'15).

[14] Tianxiang Li, Wentao Shang, Alex Afanasyev, Lan Wang, and Lixia Zhang. 2018 A Brief Introduction to NDN Dataset Synchronization (NDN Sync). In MILCOM 2018 - 2018 IEEE Military Communications Conference (MILCOM). 612-618.

[15] Teng Liang, Ju Pan, and Beichuan Zhang. 2018. NDNizing Existing Applications: Research Issues and Experiences. In Proceedings of the 5th ACM Conference on Information-Centric Networking (ICN '18). 172-183.

[16] Robert Martin, Jun-Hong Cui, and Song Han. 2014. Tracer Assisted Passive Named Data Routing for Underwater Sensor Networks. In Proceedings IEEE/MTS OCEANS.

[17] Haining Mo, Zheng Peng, Zhong Zhou, Michael Zuba, Zaihan Jiang, and JunHong Cui. 2013. Coding Based Multi-hop Coordinated Reliable Data Transfer for Underwater Acoustic Networks: Design, Implementation and Tests. In IEEE GLOBECOM. IEEE GLOBECOM, Atlanta GA.

[18] Haining Mo, Zhong Zhou, Michael Zuba, Zheng Peng, Jun-Hong Cui, and Yantai Shu. 2012. Practical Coding-based Multi-hop Reliable Data Transfer for Underwater Acoustic Networks. In Proceedings of IEEE Global Telecommunications Conference (GLOBECOM).

[19] Ilya Moiseenko and Dave Oran. 2016. TCP/ICN: Carrying TCP over Content Centric and Named Data Networks. In Proceedings of the 3rd ACM Conference on Information-Centric Networking (ACM-ICN '16). 112-121.

[20] S. Sakthivel Murugan and V. Natarajan. 2010. Performance Analysis of Signal to Noise Ratio and Bit Error Rate for Multiuser Using Passive Time Reversal Technique in Underwater Communication. In 2010 International Conference on Wireless Communication and Sensor Computing (ICWCSC).

[21] Youngtae Noh, Uichin Lee, Saewoom Lee, Paul Wang, Luiz F. M. Vieira, JunHong Cui, Mario Gerla, and Kiseon Kim. 2015. HydroCast: Pressure Routing for Underwater Sensor Networks. IEEE Transactions on Vehicular Technology (2015).

[22] Craig Partridge, Samuel Nelson, Vladimir Shurbanov, Dorene Ryder, and Bishal Thapa. 2016. NDN in Large Detached Underwater Sensing Arrays. In 2016 IEEE Globecom Workshops (GC Wkshps).

[23] Zheng Peng, Li Wei, Zigeng Wang, Lei Wan, Michael Zuba, Jun-Hong Cui, Shengli Zhou, Zhijie Shi, and James O'Donnell. 2014. Ocean-TUNE UCONN Testbed: A Technology Incubator for Underwater Communication and Networking. In IEEE Underwater Communications Networking (UCOMMS). Sestri Levante, Italy.

[24] Zheng Peng, Zhong Zhou, Jun-Hong Cui, and Zhijie Shi. 2009. Aqua-Net: An Underwater Sensor Network Architecture: Design, Implementation, and Initial Testing. In Proceedings of IEEE/MTS OCEANS.

[25] Chiara Petrioli, Roberto Petroccia, Daniele Spaccini, A. Vitaletti, Tommaso Arzilli, Davide Lamanna, Alessandro Galizial, and Enrico Renzi. 2014. The SUNRISE GATE: Accessing the SUNRISE Federation of Facilities to Test Solutions for the Internet of Underwater Things. In Proceedings of 2014 Underwater Communications and Networking (UComms'14).

[26] Tamer Refaei, Jamie Ma, Sean Ha, and Sarah Liu. 2017. Integrating IP and NDN Through an Extensible IP-NDN Gateway. In Proceedings of the 4th ACM Conference on Information-Centric Networking (ICN '17). 224-225.

[27] Wentao Shang, Zhehao Wang, Alexander Afanasyev, Jeff Burke, and Lixia Zhang. 2017. Breaking Out of the Cloud: Local Trust Management and Rendezvous in Named Data Networking of Things. In 2017 IEEE/ACM Second International Conference on Internet-of-Things Design and Implementation (IoTDI). 3-14.

[28] Susmit Shannigrahi, Chengyu Fan, and Greg White. 2018. Bridging the ICN Deployment Gap with IPoC: An IP-over-ICN Protocol for 5G Networks. In Proceedings of the 2018 Workshop on Networking for Emerging Applications and Technologies (NEAT '18). 1-7.

[29] Tyler Vernon Smith, Alexander Afanasyev, and Lixia Zhang. 2017. ChronoChat on Android. URL: https://github. com/tylervernonsmith/ChronoChat-android (2017).

[30] Milica Stojanovic and James Preisig. 2009. Underwater Acoustic Communication Channels: Propagation Models and Statistical Characterization. IEEE Communications Magazine (2009), 84-89.

[31] Ren Ting, Yu-Beng Leau, Yong Jin Park, and Joe H. Obit. 2018. Enhancing the Performance of Elliptic Curve Digital Signature Algorithm (ECDSA) in Named Data Networking (NDN). In 2018 8th IEEE International Conference on Control System, Computing and Engineering (ICCSCE). 65-69.

[32] WHOI. 2009. Micro-modem Specifications. (2009)

[33] Peng Xie, Jun-Hong Cui, and Li Lao. 2006. VBF: Vector-Based Forwarding Protocol for Underwater Sensor Networks. In Proceedings IFIP Networking. Coimbra, Portugal, 228-235.

[34] Peng Xie, Zhong Zhou, Zheng Peng, Jun-Hong Cui, and Zhijie Shi. 2010. SDRT: A Reliable Data Transfer Protocol for Underwater Acoustic Sensor Networks. Ad Hoc Networks (2010), 708-722.

[35] Guanglin Xing, Yumeng Chen, Liuting He, Wanyan Su, Rui Hou, Wei Li, Chengjun Zhang, and Xiqu Chen. 2019. Energy Consumption in Relay Underwater Acoustic Sensor Networks for NDN. IEEE Access (2019), 42694-42702.

[36] Hai Yan, Zhijie Shi, and Jun-Hong Cui. 2008. DBR: Depth-Based Routing for Underwater Sensor Networks. In Proceedings IFIP Networking. 1-13.

[37] Cheng Yi, Alexander Afanasyev, Ilya Moiseenko, Lan Wang, Beichuan Zhang, and Lixia Zhang. 2013. A case for stateful forwarding plane. Computer Communications 36, 7 (2013), 779 - 791 .

[38] Yingdi Yu, Alexander Afanasyev, David Clark, KC Claffy, Van Jacobson, and Lixia Zhang. 2015. Schematizing Trust in Named Data Networking. In Proceedings of the 2Nd ACM Conference on Information-Centric Networking (ACM-ICN '15). 177-186.

[39] Yingdi Yu, Alexander Afanasyev, and Lixia Zhang. 2015. Name-based access control. Named Data Networking Project, Technical Report NDN-0034 (2015).

[40] Zhiyi Zhang, Yingdi Yu, Sanjeev Kaushik Ramani, Alex Afanasyev, and Lixia Zhang. 2018. NAC: Automating Access Control via Named Data. In MILCOM 2018 - 2018 IEEE Military Communications Conference (MILCOM). 626-633.

[41] Zhenkai Zhu and Alexander Afanasyev. 2013. Let's ChronoSync: Decentralized dataset state synchronization in Named Data Networking. In 2013 21st IEEE International Conference on Network Protocols (ICNP). 1-10. 